\title{
At the crossroads: mucosal immunology of the larynx
}

\author{
SL Thibeault ${ }^{1}$, L Rees ${ }^{2}$, L Pazmany $^{3}$ and MA Birchall ${ }^{4}$
}

The larynx sits at the crossroads between gastrointestinal and respiratory tracts. Besides its intrinsic importance in breathing, swallowing and voice production, the larynx is also exposed to unique immunological challenges. Given the propensity of chronic inflammatory conditions such as chronic laryngitis, which affects up to $20 \%$ of Western populations, it is surprising that our understanding of the immunology of this organ remains relatively limited. Recent work on the immunological architecture of the laryngeal mucosa, and its changes that result from external challenges and inflammatory conditions, provided valuable insight into the fascinating immunology of this organ. The lessons learnt from these investigations may go beyond devising improved therapy for chronic laryngeal inflammation. Establishing whether and how the laryngeal mucosa may be involved in the modulation of wider mucosal responses may provide novel routes to the treatment of inflammatory diseases of the respiratory and alimentary tracts such as asthma and inflammatory bowel disease.

\section{INTRODUCTION}

The larynx is a vitally important mucosal organ that orchestrates swallowing, breathing, coughing and, in humans, voice. Loss of a functioning airway is life threatening, as is loss of airway protection from ingested food and drink. In man, impaired voice production holds significant implications for individual health and wellness, social and occupational function; and societal productivity. ${ }^{1,2}$ Voice is a primary mode of communication. It is tied to personal identity, and is paramount to the livelihood of millions of individuals. Conservative estimates suggest that between 5 and 10\% of the United States work force have heavy occupational voice demands, ${ }^{3}$ and that approximately 28 million workers in the United States experience voice problems daily. ${ }^{4}$ The societal cost of lost work days and treatment expenses because of voice complaints in teachers alone has been estimated at $\$ 2.5$ billion annually in the United States. ${ }^{5}$ Given the impact and high incidence of chronic inflammatory conditions affecting the larynx the relative paucity of investigations into the immunology of this organ is striking.

There is evidence supporting a distinct immunological role for the larynx. ${ }^{6}$ The larynx represents the crossroads between the gastrointestinal and respiratory systems, and is the junction between the IgA-dominated upper and IgG-dominated lower airways. It is the narrowest point of the airway. A point of greatest airway turbulence where the deposition of inhaled and sometimes ingested challenges is high. The laryngeal epithelium, such as mucosal epithelia elsewhere, comprises two main layers (epithelium, lamina propria) superficial to laryngeal muscle and cartilage. These two layers each have a characteristic immunological architecture. The epithelium of the vocal fold (medial edge) is composed of stratified squamous epithelium, whereas the neighboring ventricular folds and trachea are composed of a mixture of respiratory and stratified squamous epithelium, with the proportion of the latter increasing with age. ${ }^{7}$ The mucosa is covered with a layer of mucus produced by goblet cells and glands. The amount of stored mucus, and expression of various mucin genes, is altered in those with chronic airway inflammation. ${ }^{8}$ Within this layer, and within the epithelium itself, resides a diverse bacterial flora. ${ }^{9}, 10$ As a result of its location, the larynx is exposed to environmental challenges on both the airways and upper gastrointestinal tract. This situation could make the larynx a crucial checkpoint in the maintenance of tolerance or activation of appropriate mucosal inflammatory responses in mucosal systems downstream, such as the lung and the gastrointestinal system. 


\section{THE IMMUNOLOGICAL ARCHITECTURE OF LARYNGEAL EPITHELIUM}

Epithelial cells, coated with mucus, present a physical barrier to insults from the laryngeal lumen. However, the expression of antigen-presenting molecules on the epithelial cells highlights that they are likely to be more than inert building blocks in a wall between organism and environment.

HLA class I molecules, in humans HLA A, B, and C, sample the intracellular environment and present pathogen derived and tumor antigens. Owing to this central role in the recognition of infectious agents or malignant transformations practically all nucleated cells in the body express them. Thus, the presence of these molecules on laryngeal epithelial cells is hardly surprising. However, a detailed analysis of their expression indicated that the density of MHC class I molecules decreases toward the lumen. Intriguingly, the levels of $\beta 2$-microglubulin, a nonpolymorphic protein that is essential for the formation of functional MHC class I complexes, showed a much less pronounced reduction in the superficial layers of the epithelium. This discrepancy led to the discovery of the expression of the nonclassical MHC class I molecule CD1d on laryngeal epithelial cells. The observed gradual transition between the MHC class ${ }^{\text {hi }} \mathrm{CD} 1 \mathrm{~d}^{\mathrm{lo}}$ deeper layers to an $\mathrm{MHC}$ class $\mathrm{I}^{\text {lo }} \mathrm{CD} 1 \mathrm{~d}^{\text {hi }}$ superficial layer is an entirely unique feature of the laryngeal epithelium and suggests an interesting and potentially important compartmentalization of immune responses (Figure 1). Although the high density of MHC class I molecules in the deeper layers provides a sensitive detection for viral infections, the role of the superficial CD1drich layer is more difficult to discern. It is well documented that CD1d presents glycolipid compounds, and potentially other molecules with a lipid side chain, to a subset of lymphocytes. However, the nature of the molecules that interact with CD1d in vivo remains unclear. On one hand, there is evidence for the existence of endogenously derived self-antigens-especially in activated dendritic cells (DCs) - but the molecular characterization of these is still subject to heated debates. However, the available experimental data strongly suggests that these endogenous ligands play a key role in maintaining self-tolerance. Moreover, a number of bacterially derived CD1d antigens have been chemically identified. Whether these bacterially derived compounds contribute to the initiation of protective immunity against the pathogens that carry them or play a role in regulating self-reactivity, remains to be resolved. These facts make the interpretation of the role of the CD1d on laryngeal epithelial cells difficult. Does the superficially placed CD1d expression represent a pathway that is designed to maintain tolerance toward the constant onslaught of inhaled and swallowed antigens? It could be argued that in the absence of such protective mechanism anyone not breathing HEPA-filtered air and drinking double distilled water would suffer from a constant laryngeal inflammation. However, it is equally plausible that the presence of CD1d plays a role in monitoring the bacterial content of the larynx and represents an early, relatively nonspecific, warning mechanism for the detection of changes in the flora.

The other tantalizing feature of antigen presentation in the larynx is the presence of HLA class II molecules. Investigations on laryngeal mucosal biopsies taken from the false vocal fold of healthy individuals found that laryngeal epithelial cells express MHC class II antigen-presenting molecules distributed uniformly throughout the entire thickness of the laryngeal epithelium in vivo. ${ }^{11}$ These molecules, involved in the presentation of exogenous antigens to $\mathrm{CD} 4{ }^{+}$"helper" $\mathrm{T}$ cells, are typically only present on professional antigen-presenting cells, such as DC, monocytes/macrophages, although cytokine exposure can induce MHC class II expression in other cells. Normal laryngeal epithelial cells cultured in vitro did not

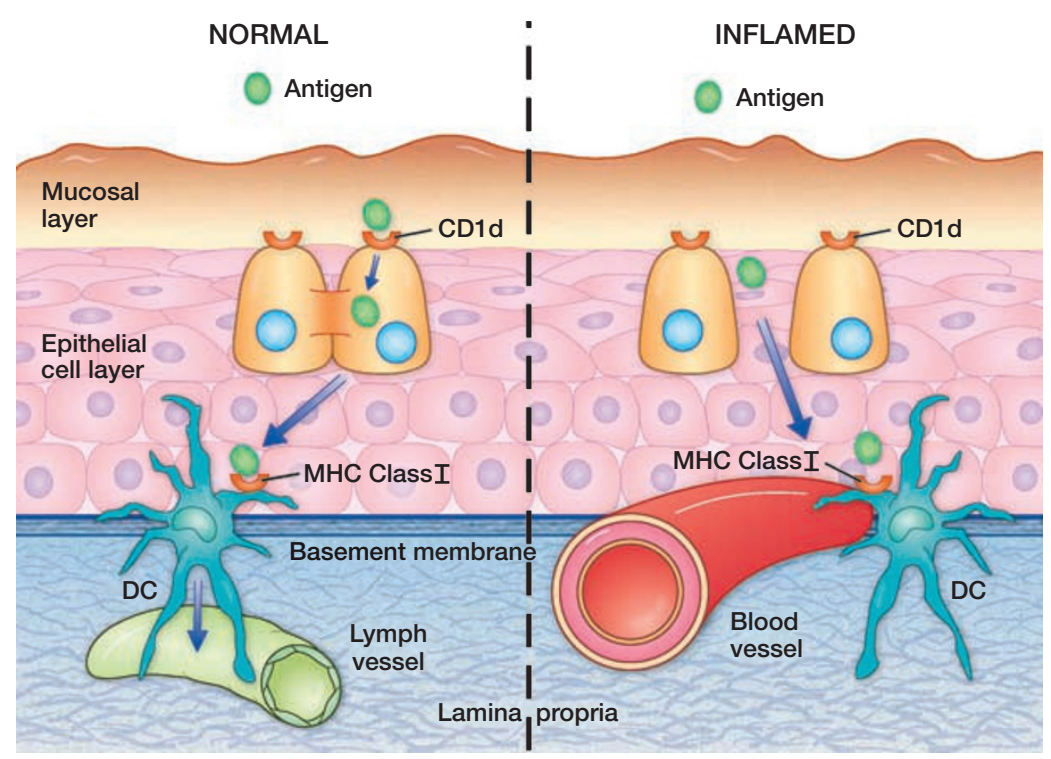

Figure 1 The laryngeal mucosa is divided into two layers in terms of expression of antigen-presenting molecules by epithelial cells: a superior, CD1d-rich layer and deep, MHC class I-rich layer. We hypothesize that challenges that break down intercellular adhesion permit the presentation of incident antigens directly to the more "proinflammatory" deeper layer, resulting in chronicity. Dendritic cells straddle the basement membrane and may orchestrate this response. 
constitutively express MHC class II molecules. However, stimulating these cultures with interferon- $\gamma$ restored the transcription of these antigen-presenting molecules to levels seen in the biopsy specimens. ${ }^{12}$ The fact that the participants in this study did not have any clinical or histological evidence of inflammation raises the question: what is the mechanism that induces the expression of MHC class II molecules in the larynx in vivo? Is this phenomenon because of a basal cytokine secretion elicited by the presence of the bacterial flora or is there a particular cell type in the immune cell compartment present in the epithelial and submucosal layers that constitutively secrete IFNg and/or other cytokines?

As mentioned above, the expression of MHC class II molecules is primarily a "privilege" of antigen-presenting cells. On the surface of DC, monocytes and macrophages antigens bound to class II proteins would be accompanied by so-called co-stimulatory molecules, such as CD80 and CD86, and the coexistence of these two signals would result in the antigen-specific T-cell responses. In contrast, MHC class II bound antigens in the absence of co-stimulation results in tolerance induction. Thus, the outcome of this antigen presentation pathway on the surface of laryngeal epithelial cells is to a large extent dependent on the presence of absence of co-stimulatory molecules. Although no data has been reported on this issue in the literature, it is tempting to speculate that class II-mediated antigen presentation in the absence of CD80/CD86 could be a simple and effective mechanism to induce tolerance toward inhaled, potentially immunogenic, compounds that have the capacity to bind to MHC class II molecules. However, although this assumption is entirely plausible, the fact that the laryngeal epithelium in some species, for example pigs, is devoid of MHC class II molecules may question the importance of this pathway in tolerance induction.

\section{HAEMOPOIETIC CELLS INTHE LARYNX}

Cell types identified in the laryngeal mucosa and associated with changes in challenge states in adult mammals include macrophages, DC, T and B lymophocytes and NK cells, as well as granulocytes and macrophages. ${ }^{6,13-15}$ Highly organized lymphoid collections, similar to Peyer's patches (laryngealassociated lymphoid tissue $)^{16-19}$ have been identified by postmortem serial section studies in $80 \%$ of persons younger than 20 years of age and $56 \%$ of persons older. ${ }^{16,18}$ These structures are rare below the epiglottis. ${ }^{18}$ However, similarly to the small intestine, more than $90 \%$ of immune cell population of the larynx is distributed in diffuse manner throughout the epithelium and lamina propria. ${ }^{20} \mathrm{DC}$, NK cells, and T and B cells are the most frequent in the subglottis and rarest in the vibratory vocal-fold area whereas granulocytes and macrophages are evenly distributed throughout the whole laryngeal mucosa. In human fetal larynges, DC, T and B cells, and macrophages are present in the ventricular folds and subglottis, but not the glottis. ${ }^{21} \gamma \delta$-T cells, which are often referred to as "intraepithelial lymphocytes" and appear to be important in local responses in the skin and bowel, are very rarely observed in any layer in the larynx.
Owing to their pivotal role in the initiation of immune responses, the DC compartment of the laryngeal tissues has been the subject of multiple studies. In a pig model of laryngeal transplantation, a dense population of DC particularly around the basement membrane has been observed. ${ }^{22}$ Furthermore, the activation status of these cells changed following reperfusion. Dendrites from this area stretch through the epithelium and are likely to contact the superficial mucosal layer that also contains some elements of the bacterial flora. ${ }^{6}$ Similarly to the observations in pigs, a dense population of DC is present in the human laryngeal epithelium. ${ }^{11}$

The analysis of various lymphocytes subsets and their relationship to the antigen-presenting molecules within the mucosa has seen major advances recently, because of a technical development. Previously, analysis of cellular interactions in situ has relied on the observer examining overlapping cells and assessing whether an interaction was taking place. This is lengthy, subject to inter- and intra-operator variation and gives no indication of whether cells are associating randomly or preferentially as the probability of these "interactions" increases with the number of cells. Analysis by eye is also insufficiently sensitive to utilize all the data generated. A method for automated, pixel-based analysis of digital immunofluorescent images has been developed. ${ }^{23,24}$ A macro written within the freely available image analysis program Image ${ }^{25}$ counts the number of pixels of each color, and then displays them as proportions of the positive area. Although this analysis does involve some data loss, it provides an objective assessment of the amount of staining and permits statistical analysis of the levels of cellular colocalization. By calculating the expected levels of colocalization within the tissues if cells associate randomly and comparing this to the observed levels of colocalization, it is possible to determine whether overlapping between different cell types is occurring at significantly greater levels than random chance would predict. Increases in colocalization indicate prolonged cell contact; therefore, it is possible to gain some information about the dynamic interactions between cells from static tissue sections. This approach was used to evaluate the presence of $\mathrm{CD} 4^{+}$and $\mathrm{CD} 8^{+} \mathrm{T}$ cells as well as B lymphocytes. The results have shown an increased number of both $\mathrm{T}$ and $\mathrm{B}$ cells in the lamina propria, compared to the epithelium, and the almost complete absence of $B$ lymphocytes of the epithelial layer. Despite significant interindividual variability, this study demonstrated the preferential localization of $\mathrm{CD}^{+} \mathrm{T}$ lymphocytes to the deeper, $\mathrm{MHC}$ class $\mathrm{I}^{\text {high }}$ layers of the epithelium. As these T cells recognize antigens presented by MHC class I molecules, these results appear logical and provide validation for the digital image analysis approach. In the course of this work, the accumulation of $\mathrm{CD}^{+} \mathrm{CD} 161^{+}$ lymphocytes was seen in the upper, $\mathrm{CD} 1 \mathrm{~d}^{\text {high }}$ layer of the epithelium. The coexpression of these two markers was the first definition of Natural T (NKT) cells, which are sometimes also referred to as Natural Killer T lymphocytes. Although this definition of NKT cells has now been superseded-some $\mathrm{CD}^{+}{ }^{+} \mathrm{CD} 161^{+}$ lymphocytes are not true NKT cells whereas some NKT cells do not express CD161 - the colocalization of these double-positive cells with CD1d antigen-presenting molecules provides very 
strong indirect evidence for the presence of NKT cells in the superficial mucosa. NKT cells play a role in the control of some bacterial infections and show direct and indirect cytotoxicity against certain tumor cells. Just as important, in animal models, the elimination of this lymphocyte subset resulted in the breakdown of self-tolerance. Thus, their presence in the laryngeal epithelium could be consistent with a tolerance-inducing function. This makes additional experiments for the confirmation of NKT cell presence paramount. However, currently, the lack of suitable reagents limits the use of immunofluorescence for these investigations.

\section{THE EFFECTS OF EXTERNAL CHALLENGES ON LARYNGEAL MUCOSAL IMMUNE ARCHITECTURE Bacteria}

The importance of the bacterial flora in the development of the normal immunological architecture of the gut has been studied rather extensively (for a recent review see Rees et al. ${ }^{11}$ ). In the larynx, a similar complex interaction between the bacterial flora, the expression of antigen-presenting molecules and the lymphocyte/monocyte compartment has been investigated in animal models. In the most extensive study, 23 piglets were delivered by Cesarian section into a germ-free "bubble". The laryngeal mucosa from two animals killed on day 0 showed very few $\mathrm{CD}^{+} \mathrm{T}$ cells and no expression of CD16, a marker of activated DC, or MHC class II molecules. As laryngeal epithelial cells in pigs are devoid of class II molecules, the absence of this marker in these animals indicates the lack of monocytes, DC, and activated $\mathrm{T}$ and $\mathrm{B}$ lymphocytes. The remaining animals were divided into three groups. Some were kept germ free, the second group was colonized by a standard bacterial flora (modified Schaedler) and additional bacillus species whereas the third group received the same standard flora supplemented with staphylococci. At week 3 germ-free animals showed little increase in the expression of the above-described three markers compared to birth. In contrast, all colonized animals developed a rich $\mathrm{CD} 4^{+}$cell population, which was more extensive in animals also carrying staphylococci. Importantly, animals colonized with the mixture including staphylococci, a normal commensal organism in pigs, were the only group to show an increase in MHC II expression, indicating the presence of a significant monocyte, dendritic cell, activated lymphocyte population. ${ }^{26}$ Similarly, in rats, the introduction of heat inactivated Moraxella or Bordetella resulted in the recruitment granulocytes, DC, and various lymphocyte subsets. ${ }^{14}$ These experiments clearly demonstrated that the development of laryngeal mucosal immune architecture depends on interaction with bacteria, and that the composition of the bacterial flora significantly influences the recruitment of leukocytes to the mucosa. On the basis of these observations, it is reasonable to propose that seemingly minor changes of the commensal flora could significantly alter the immunological environment in the larynx. In turn. these changes could play a key role in the development of chronic inflammatory states. Unfortunately, our understanding of the normal bacterial flora in the larynx is currently somewhat limited. ${ }^{27}$ Most studies in this field have concentrated on the presence of Helicobacter pylori; however, the conclusions of this body of work show some contradiction. One group detected no $H$. pylori-like organisms in laryngeal samples using Giemsa and hematoxylin-eosin staining, ${ }^{28}$ whereas another study using similar techniques detected organisms resembling Helicobacter, although these bacteria could not be positively identified by immunohistochemistry. ${ }^{29}$ In contrast Fang et al.,${ }^{30}$ Rubin et al. ${ }^{31}$ Borkowski et al., ${ }^{32}$ and Jaspersen et al., ${ }^{33}$ all report the presence of $H$. pylori via urease assays and histology in patients with benign laryngeal lesions or chronic laryngitis.

\section{Tobacco smoke}

Studies on the immunological consequences of tobacco challenges have repeatedly shown changes in the laryngeal mucosa immune response. In one recent study, the density of immunologically relevant cells in false vocal-fold biopsies were examined in a cohort of 63 individuals, both smokers and nonsmokers, between the ages of $18-80$ years, with no history of laryngeal or pharyngeal cancer or other obvious pathology. The presence of lymphocytes ${ }^{34}$ in the laryngeal mucosa of current cigarette smokers showed increased numbers of $\mathrm{CD} 4^{+} \mathrm{T}$ cells and there was an association between older age and greater $\mathrm{CD} 4^{+} \mathrm{T}$-cell numbers in both epithelium and lamina propria. Older age and female gender were associated with decreased lamina propria $\mathrm{CD} 4{ }^{+} \mathrm{CD} 45 \mathrm{RO}^{+} \mathrm{T}$ cells and an increase in $\mathrm{CD} 4{ }^{+} \mathrm{CD} 45 \mathrm{RO}^{-} \mathrm{T}$ cells, indicating the relative paucity of memory $\mathrm{T}$ cells in these particular patients. In a related study of patients with hoarseness, however, DNA microarray analysis of laryngeal polyps did not detect differential expressed transcripts between smokers compared to nonsmokers. However, as only 7 of the 28 patients recruited were current nonsmokers, the power of the study was limited, potentially explaining the lack of a recognizable gene expression signature associated with smoking. ${ }^{35}$ In addition, a considerable number of genes upregulated in polyps were of immunological relevance including, but not limited to-the HLA class II molecules HLA-DRA and HLA DPB1, RFX5-a component of the promoter of MHC class II genes and monocyte-macrophage differentiation protein. A pilot study measuring antigen-presenting molecule expression in Reinke's edema (a benign inflammatory bilateral lesion of the membranous vocal folds, common in smokers), demonstrated a dense population of MHC class $\mathrm{II}^{+}$and $\mathrm{CD} 68^{+}$cells (Figure 2). Such dual positive cells are likely to be activated macrophages and theoretically cytokine secretion by these, could explain the already mentioned changes in $\mathrm{CD}_{4}{ }^{+} \mathrm{T}$-cell numbers in smokers. However, validating this assumption, and elucidating the mechanisms that lead to the appearance of these activated macrophages in the mucosa of smokers will require further work.

\section{Extra-esophageal reflux}

Extra-esophageal reflux (also referred to as posterior laryngitis or laryngo-pharyngeal reflux, LPR) is a very common condition, affecting an estimated $20 \%$ of the adult US population. ${ }^{36}$ This disease is costly in terms of both health-care expenditure and quality of life. Over 200 million is spent annually in the United States on proton pump inhibitors, ${ }^{37}$ the most common 

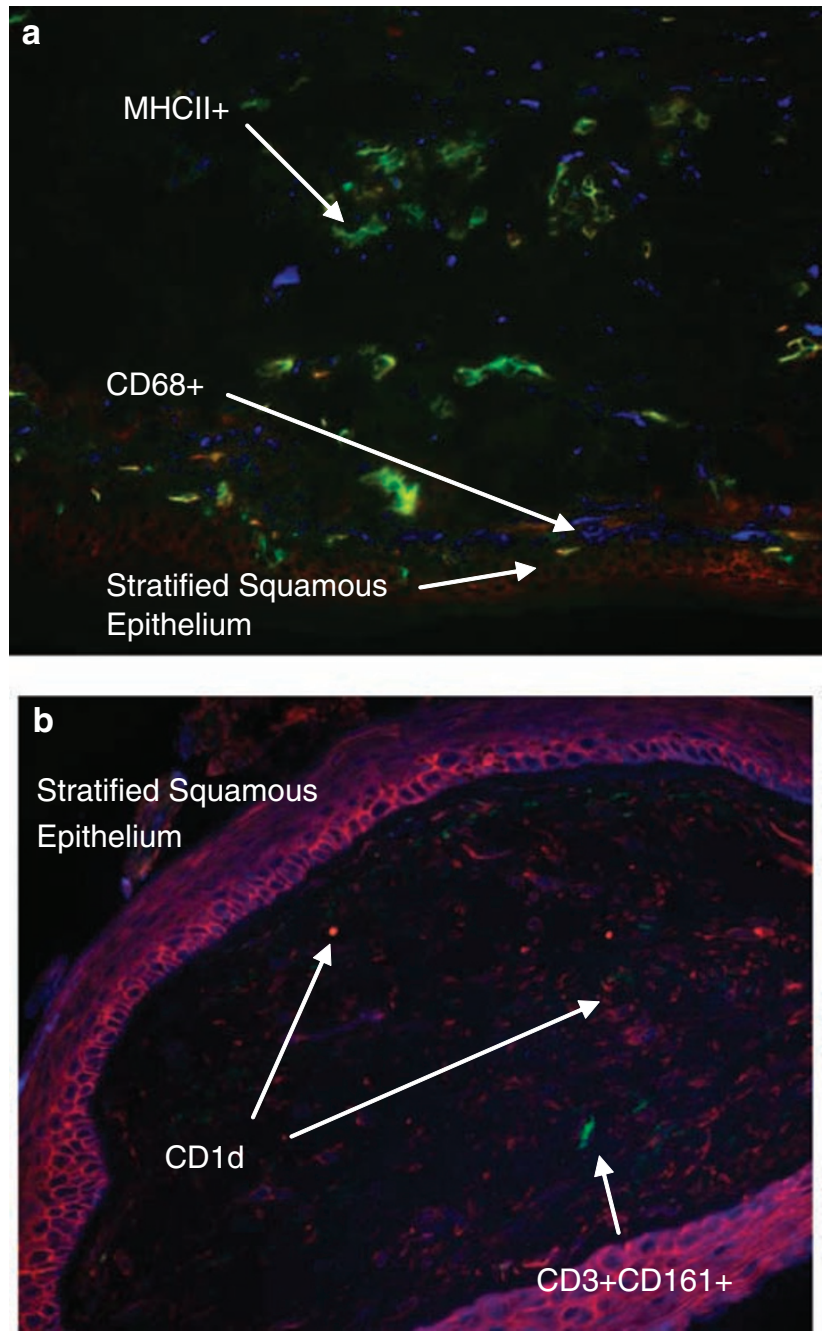

Figure 2 Immunofluorescence images of Reinke's edema. (a) Dense population of lamina propria by MHCll + (green) and CD68+ (blue) cells (b) strong membranous expression of CD1d (red) by epithelial and dendritic cells, and increased CD3 + CD161 + (NKT) cells in lamina propria (blue-green).

medical management for LPR. The main clinical features are chronic mucosal inflammation, including edema and erythema. Globus pharyngeus and chronic cough are symptoms frequently associated with the condition. In patients suffering from LPR, the larynx is repeatedly exposed to gastric contents. This chronic challenge could conceivably alter the immunological responses. In a study by Rees et al., ${ }^{38} 12$ patients with florid LPR were compared to 11 control individuals who did not suffer from the condition. Diagnosis was based on validated clinical scoring, ${ }^{39}$ dual-probe $\mathrm{pH}$ studies, transnasal esophagoscopy, and manometry. Multiple color immunofluorescence studies of biopsies taken from the posterior (nonvibratory) vocal fold detected a strongly statistically significant increase in $\mathrm{CD} 8^{+}$ $T$ cells numbers in the laryngeal epithelium of LPR sufferers. It is noteworthy that, despite the change in the numbers of the cytotoxic T cells-which recognize MHC class I restricted antigens, there was no detectable change in the density of the antigen-presenting molecules themselves on the epithelial cells. ${ }^{38}$
The expression of MHC class II molecules was also unchanged in LPR patients. However, there was a marked increase in the presence of the nonclassical MHC molecule CD1d in the patient group and this coincided with an elevated number of $\mathrm{T}$ cells colocalizing with the CD1d-expressing epithelial cells. As discussed before, currently the lack of reagents hinders the positive identification of NKT cells that recognize antigens presented via CD1d. However, by definition NKT cells specifically recognize antigens via this pathway, and currently there is no evidence for other lymphocyte subsets binding to this molecule. Thus, based on the available data, it seems safe to conclude that in LPR elevated CD1d levels lead to increased NKT cell contact with the superficial layers of the laryngeal epithelium. This situation raises three questions: (i) what is the mechanism that leads to the upregulation of CD1d expression; (ii) what antigens are being presented by CD1d in LPR, and (iii) is the observed upregulation protective, or detrimental in terms of the inflammatory response seen in the disease? Obviously, in the absence of experimental data, we can only speculate on the answers. However, recent work has identified a number of structural features that determine whether CD1d-restricted antigens are recognizable by the T-cell receptors on NKT cells or not. ${ }^{40}$ This work highlights two interesting facts. First, the likelihood of bacterial compounds binding to CD1d seems higher than previously appreciated. Thus, relatively subtle changes in the laryngeal flora may have a significant effect on CD1d-mediated antigen presentation. Perhaps even more interesting, it appears that the chemical modification of the sugar components of CD1d-bound antigens profoundly influence their ability to interact with the T-cell receptor. It is tempting to speculate that the presence of the acidic refluxate in LPR may result in such chemical changes. These alterations could potentially "inactivate" previously tolerance inducing CD1d antigens, leading to the loss of their "immunosuppressive" properties. Alternatively, the chemical modification may enhance the NKT cell-mediated recognition of otherwise innocuous CD1d-binding bacterial compounds, resulting in a productive immune response. Obviously, either of these mechanisms could explain the development of the chronic inflammations seen in the disease.

\section{DOES THE LARYNGEAL MUCOSA INFLUENCE LOWER AIRWAY IMMUNE RESPONSES?}

The larynx is positioned at an immunological and anatomical crossroad. It represents the junction between the sterile, IgGdominated lower airway, and the bacteria-rich, IgA-dominated upper airway. Everything we inhale passes through its turbulent, narrow aperture in "breathing mode" and everything we ingest passes over its surface in "sphincter mode" (Figure 3). Teleologically, it seems necessary to place a tolerance-inducing mechanism here that would protect against sensitization to inhaled and ingested compounds, which could otherwise act as antigens. Furthermore, the effective functioning of this tolerance induction could protect both the downstream respiratory tract and the gastrointestinal system from potentially harmful, and debilitating sensitization. Thus, the larynx would be an ideal point to place a mucosal immune checkpoint. However, 


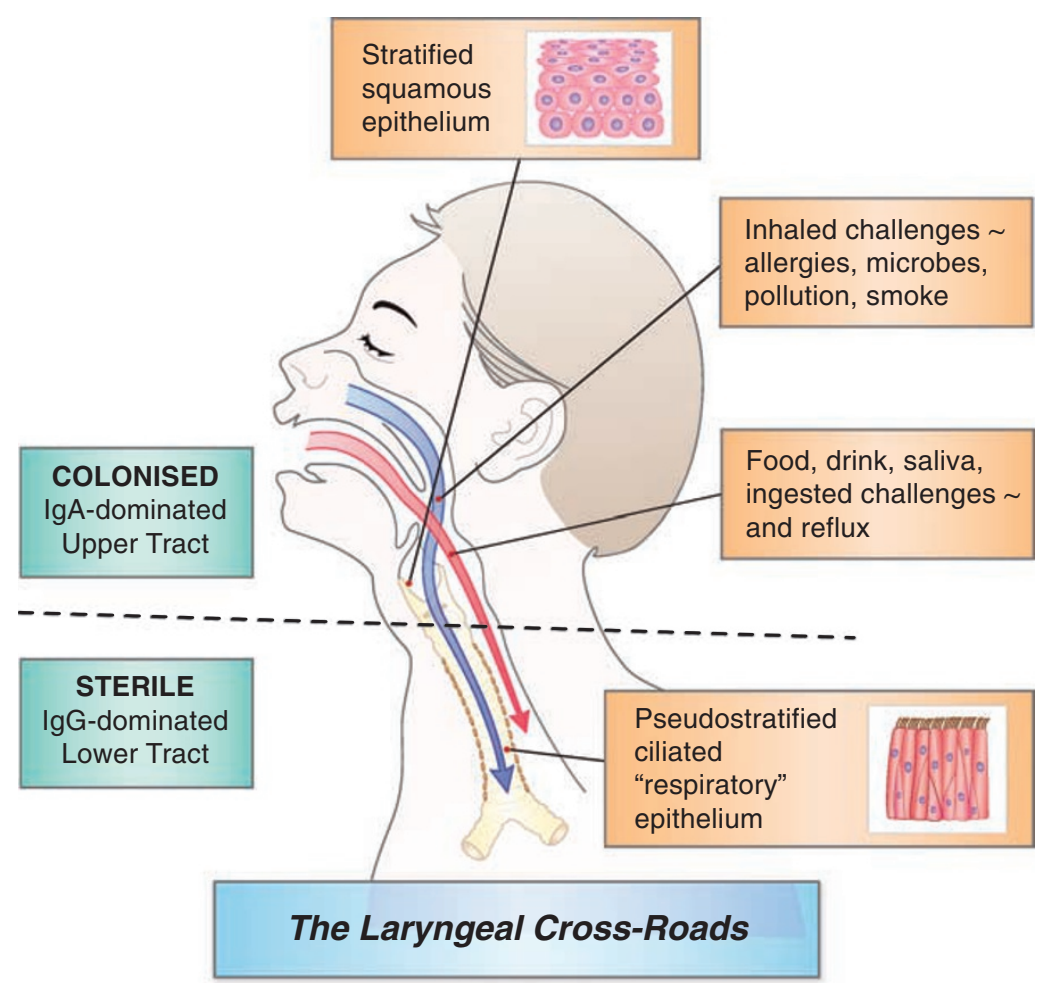

Figure 3 The laryngeal crossroads represents the junction between the sterile, IgG-dominated lower airway and the bacteria-rich, IgA-dominated upper airway. Things inhaled pass through a turbulent, narrow aperture in "breathing mode" and everything ingested passes over the larynx's surface in "sphincter mode".

despite the innate attractiveness of this paradigm, presently there is only tenuous circumstantial evidence to support it. As presented, the larynx possesses a sophisticated immune architecture that alters significantly in response to a wide variety of incident challenges. Cell type and colocalization studies suggest that laryngeal mucosa is equipped with antigen presentation and cellular pathways that could fulfill the proposed protective role. It is widely observed, although poorly quantified, that patients who have had a total laryngectomy suffer increased chest and other infections, ${ }^{41}$ also suggesting a wider immune role for this organ. It could be argued that inhaled steroids and other medications for asthma exert their therapeutic effect via the larynx, asa particles are likely to be deposited in much higher densities on the larynx than more distally in the respiratory tree. Studies to establish the exact place of the larynx fits in the hierarchy of respiratory mucosal immunity are hampered by the lack of a suitable animal model, as it is almost impossible to separate immunological challenges of the upper and lower airways in small animals and appropriate conditional and transgenic mice are lacking. Nonetheless, this question remains the most pressing in the field of laryngeal biology, and demands detailed investigation. The possible implications of a better understanding of laryngeal immunology for the way we treat respiratory disease could be far reaching.

\section{CONCLUDING REMARKS}

In summary, the laryngeal epithelium has a rich immunological architecture, with molecular and cellular pathways that could control unwanted immune responses against harmless everyday challenges. Whether such responses actually take place, and mechanism involved in them, remain currently unknown. Investigations of the immunological basis of chronic inflammation of the larynx are of fundamental importance to making progress in our understanding, and thereby treatment, of the millions of people with laryngeal symptoms each year. In addition, clarification of the size and type of contribution of the larynx to respiratory and systemic immunity is required. It may very well be that the larynx, the caretaker of the anatomical crossroads, also plays a similar central role in the aerodigestive mucosal immunity. Improving our understanding of the immunological responses of the laryngeal mucosa may have far reaching implications for the future management of diseases as diverse as asthma, food allergy, pseudocroup, chronic cough, and esophagitis.

\section{DISCLOSURE}

The authors declared no conflict of interest.

(C) 2009 Society for Mucosal Immunology

\section{REFERENCES}

1. Jacobson, B.H., Johnson, A. \& Grywalski, C. The voice handicap index (VHI): development and validation. Am. J. Speech Lang. Pathol. 6, 66-70 (1997).

2. Ma, E.P. \& Yiu, E.M. Voice activity and participation profile: assessing the impact of voice disorders on daily activities. J. Speech Lang. Hear. Res. 44, 511-524 (2001). 
3. Roy, N. et al. Three treatments for teachers with voice disorders: a randomized clinical trial. J. Speech Lang. Hear. Res. 46, 670-688 (2003).

4. Verdolini, K. \& Ramig, L. Review: occupational risks for voice problems. Logoped. Phoniatr. Vocol. 26, 37-46 (2001).

5. Coyle, S.M., Weinrich, B.D. \& Stemple, J.C. Shifts in relative prevalence of laryngeal pathology in a treatment-seeking population. J. Voice 15, 424-440 (2001).

6. Barker, E., Haverson, K., Stokes, C.R., Birchall, M. \& Bailey, M. The larynx as an immunological organ: immunological architecture in the pig as a large animal model. Clin. Exp. Immunol. 143, 6-14 (2006).

7. Stell, P.M., Stell, I.M. \& Watt, J. Age changes in the epithelial lining of the human larynx. Gerontology 28, 208-214 (1982).

8. Innes, A.L. et al. Epithelial mucin stores are increased in the large airways of smokers with airflow obstruction. Chest 130, 1102-1108 (2006).

9. Gillet, S. et al. Molecular identification of the bacterial flora of the larynx. Clin. Otolaryngol. (in press).

10. Gillet, S., Rees, L.E., Coogan, T., Birchall, M. \& Bailey, M. Characterization of the bacterial flora of the larynx. Clin. Otolaryngol. 31, 583 (2006).

11. Rees, L.E., Ayoub, O., Birchall, M.A., Haverson, K. \& Bailey, M. Quantiatitive immunofluorescence to measure MHC Class II expression on laryngeal epithelium. Clin. Exp. Immunol. 134, 497-502 (2003)

12. Rees, L.E., Gunasekaran, S., Sipaul, F., Birchall, M.A. \& Bailey, M. The isolation and characterisation of primary human laryngeal epithelial cells. Mol. Immunol. 43, 725-730 (2006).

13. Jecker, P., Ptok, M., Pabst, R. \& Westermann, J. Distribution of immunocompetent cells in various areas in the normal laryngeal mucosa of the rat. Eur. Arch. Otorhinolaryngol. 253, 142-146 (1996).

14. Jecker, P. et al. Acute laryngitis in the rat induced by Moraxella catarrhalis and Bordetella pertussis: number of neutrophils, dendritic cells, and $\mathrm{T}$ and $\mathrm{B}$ lymphocytes accumulating during infection in the laryngeal mucosa strongly differs in adjacent locations. Pediatr. Res. 46, 760-766 (1999).

15. Jecker, P., Mann, W.J., McWilliam, A.S. \& Holt, P.G. Dendritic cell influx differs between the subglottic and glottic mucosae during acute laryngotracheitis induced by a broad spectrum of stimuli. Ann. Otol., Rhinol. Laryngol. 111, 567-572 (2002)

16. Debertin, A.S. et al. Coincidence of different structures of mucosaassociated lymphoid tissue (MALT) in the respiratory tract of children: no indications for enhanced mucosal immunostimulation in sudden infant death syndrome (SIDS). Clin. Exp. Immunol. 146, 54-59 (2006).

17. Kracke, A. et al. Larynx-associated lymphoid tissue (LALT) in young children. Anat. Rec. 248, 413-420 (1997)

18. Hiller, A.S., Tschernig, T., Kleemann, W.J. \& Pabst, R. Bronchusassociated lymphoid tissue (BALT) and larynx-associated lymphoid tissue $(\mathrm{LALT})$ are found at different frequencies in children, adolescents and adults. Scand. J. Immunol. 47, 159-162 (1998).

19. Kutta, H., Steven, P., Tillmann, B.N., Tsokos, M. \& Paulsen, F.P. Regionspecific immunological response of the different laryngeal compartments: significance of larynx-associated lymphoid tissue. Cell Tissue Res. $\mathbf{3 1 1}$ 365-371 (2003)

20. Rees, L.E. et al. Lifestyle factors influence immunology architecture of the human larynx. Clin. Immunol. 118, 324-327 (2006).

21. Dietrich, C., Jecker, P., Tschernig, T. \& Mann, W.J. Presence of dendritic cells, T lymphocytes, macrophages, B lymphocytes and glandular tissue in the human fetal larynx. Acta Otolaryngologica 124, 833-838 (2004).

22. Barker, E. et al. Early immunological changes associated with laryngeal transplantation in a major histocompatibility complex-matched pig model. Clin. Exp. Immunol. 146, 503-508 (2006).

23. Inman, C.F. et al. Validation of computer-assisted, pixel-based analysis of multiple-colour immunofluorescence histology. J. Immunol. Methods 302, 156-167 (2005).

24. Inman, C., Bailey, J.C., Cook, S. \& Bailey, M. Interactions between immune cells and their microenvironment. Vet. Immunol. Immunopathol. 120, 10-19 (2007).

25. http://rsb.info.nih.gov/ij/.

26. Birchall, M. et al. The development of upper airway mucosal immune architecture depends on peri-natal bacterial colonisation. Clin. Otolaryngol. 33, 299 (2008).

27. Tanaka, I., Suzuki, K., Tanaka, E. \& Baba, S. Investigation of normal bacterial flora in the upper respiratory tract. Acta Otolaryngologica, Supplement 525, 44-50 (1996).

28. Kizilay, A. et al. Histopathologic examination for Helicobacter pylori as a possible etiopathogenic factor in laryngeal carcinoma. Chemotherapy 52 , 80-82 (2006).

29. Akbayir, N., Basak, T., Seven, H., Sungun, A. \& Erdem, L. Investigation of Helicobacter pylori colonization in laryngeal neoplasia. Eur. Arch. Otorhinolaryngol. 262, 170-172 (2005).

30. Fang, T.J., Lee, L.A., Li, H.Y., Yang, C. \& Huang, C.G. Helicobacter pylori colonization in the larynges of patients with hoarseness. Laryngoscope 118, 389-393 (2008)

31. Rubin, J.S., Benjamin, E., Prior, A., Lavy, J. \& Ratcliffe, P. The prevalence of Helicobacter pylori infection in benign laryngeal disorders. J. Voice 16, 87-91 (2002)

32. Borkowski, G. et al. A possible role of Helicobacter pylori infection in the etiology of chronic laryngitis. Eur. Arch. Otorhinolaryngol. 254, 481-482 (1997).

33. Jaspersen, D. et al. Is chronic laryngitis associated with Helicobacter pylori? Results of a prospective study. Z. Gastroenterol. 36, 369-372 (1998).

34. Rees, L.E. et al. Smoking influences the immunological architecture of the human larynx. Clin. Immunol. 118, 342-347 (2006).

35. Duflo, S. et al. Differential gene expression profiling of vocal fold polyps and Reinke's edema by cDNA microarray. Ann. Otol., Rhinol. Laryngol. 115, 703-714 (2006)

36. Koufman, J.A., Amin, M.R. \& Panetti, M. Prevalence of reflux in 113 consecutive patients with laryngeal and voice disorders. Otolaryngol. Head Neck Surg. 123, 385-388 (2000).

37. Karkos, P.D. \& Wilson, J.A. Empiric treatment of laryngopharyngeal reflux with proton pump inhibitors: a systematic review. Laryngoscope 116, 144-148 (2006).

38. Rees, L.E. et al. The mucosal immune response to laryngopharyngeal reflux. Am. J. Respir. Crit. Care Med. 177, 1187-1193 (2008).

39. Belafsky, P.C., Postma, G.N., Amin, M.R. \& Koufman, J.A. Symptoms and findings of laryngopharyngeal reflux. Ear Nose Throat J. 81, 10-13 (2002).

40. Tonti, E. et al. NKT cell help to B lymphocytes can occur independently of cognate interaction. Blood (in press).

41. Fontana, G.A. et al. Coughing in laryngectomized patients. Am. J. Respir. Crit. Care Med. 160, 1578-1584 (1999). 